\title{
Dropwise condensation on hydrophobic bumps and dimples
}

\section{Citation}

Yao, Yuehan, Joanna Aizenberg, and Kyoo-Chul Park. 2018. “Dropwise Condensation on Hydrophobic Bumps and Dimples." Applied Physics Letters 112 (15) (April 9): 151605. doi: $10.1063 / 1.5021343$.

\section{Published Version}

doi:10.1063/1.5021343

\section{Permanent link}

http://nrs.harvard.edu/urn-3:HUL.InstRepos:36642002

\section{Terms of Use}

This article was downloaded from Harvard University's DASH repository, and is made available under the terms and conditions applicable to Open Access Policy Articles, as set forth at http:// nrs.harvard.edu/urn-3:HUL.InstRepos:dash.current.terms-of-use\#OAP

\section{Share Your Story}

The Harvard community has made this article openly available.

Please share how this access benefits you. Submit a story.

Accessibility 


\title{
Dropwise Condensation on Hydrophobic Bumps and Dimples
}

\author{
Yuehan Yao ${ }^{1}$, Joanna Aizenberg ${ }^{2,3}$, Kyoo-Chul Park ${ }^{4 *}$ \\ ${ }^{1}$ Department of Materials Science and Engineering, Northwestern University, Evanston, IL 60208, USA; \\ ${ }^{2} J o h n$ A. Paulson School of Engineering and Applied Sciences, Harvard University, Cambridge, MA 02138, \\ USA; \\ ${ }^{3}$ Wyss Institute for Biologically Inspired Engineering, Harvard University, Cambridge, MA 02138, USA; \\ ${ }^{4}$ Department of Mechanical Engineering, Northwestern University, Evanston, IL 60208, USA.
}

\begin{abstract}
Surface topography plays an important role in promoting or suppressing localized condensation. In this work, we study the growth of water droplets on hydrophobic convex surface textures such as bumps and concave surface textures such as dimples with a millimeter scale radius of curvature. We analyze the spatio-temporal droplet size distribution under a supersaturation condition created by keeping the uniform surface temperature below dew point, and show its relationship to the sign and magnitude of the surface curvature. In particular, in contrast to the well-known capillary condensation effect, we report an unexpectedly less favorable condensation on smaller, millimeter-scale dimples where capillary condensation effect is negligible. To explain these experimental results, we numerically calculated the diffusion flux of water vapor around the surface textures, showing that its magnitude is higher on bumps and lower on dimples compared to a flat surface. We envision that our understanding of millimetric surface topography can be applied to improve the energy efficiency of condensation in applications such as water harvesting, heating, ventilation, and air conditioning (HVAC) systems for buildings and transportation, heat exchangers, thermal desalination plants, and fuel processing systems.
\end{abstract}


Controlling condensation and droplet growth is fundamentally important in a wide spectrum of industrial applications including water harvesting from thin air, multi-stage flash desalination, heat exchangers, and thermal power generation. ${ }^{1-5}$ Extensive research efforts have been devoted to promote dropwise condensation of water by employing various surface chemistries and micro-/nano-textures. ${ }^{2-10}$ While recent studies have shown that droplet growth on millimetric convex surfaces by condensation is facilitated, ${ }^{11-13}$ a more detailed study on droplet growth on textured surfaces is required to systematically understand the impact of the sign and the magnitude of the radius of curvature of surface features. Here, we provide a quantitative analysis of droplet growth dynamics on hydrophobic surfaces with a wide range of millimeter-scale surface topographies ranging from convex textures (e.g., bumps) to concave textures (e.g., dimples).

To experimentally study the dropwise condensation on textured surfaces, we used the materials and experimental setup that are similar to our previous study. ${ }^{11}$ The bump and dimple patterns were transferred from a pair of 3D printed molds (Objet30, Stratasys) by pressing a thin aluminum sheet $(63.5 \mathrm{~mm} \times 63.5 \mathrm{~mm} \times 0.127 \mathrm{~mm}$, McMaster-Carr $)$ between the two 3D printed molds. For testing the effect of surface roughness on condensation, we roughened some samples using sandpaper before transferring the bump/dimple patterns. To change the surface chemistry of the patterned samples, they were then cleaned and immersed in $1 \mathrm{wt} \%$ solution of fluoroaliphatic phosphate ester fluorosurfactant (FS-100, Pilot Chemical) in ethanol at $70^{\circ} \mathrm{C}$ for 30 minutes. Surface temperature $\left(8.5 \pm 0.4^{\circ} \mathrm{C}\right)$, ambient temperature $\left(21.2 \pm 0.2^{\circ} \mathrm{C}\right)$, and relative humidity $(70 \%$ $\pm 2 \%$ ) were carefully controlled throughout the experiments by employing a customized cooling system and a controlled-humidity chamber. See Methods and Figs. S1-S3 in Supplementary Material for more detailed information. 
Fig. 1 shows representative images of condensed droplets at $t=2,400 \mathrm{sec}(=40 \mathrm{~min})$ on textured surfaces with a radius of curvature $\left(\kappa^{-1}\right)$ varying from $-13.71 \mathrm{~mm}$ to $+13.71 \mathrm{~mm}$, where the negative sign represents a concave feature (dimple) and the positive sign represents a convex feature (bump). Qualitatively, the size of the droplets condensing at the center of dimples is noticeably smaller than that of the droplets at the apex of bumps (see insets with yellow hollow circles next to the bump/dimple images in Fig. 1), regardless of surface roughness (see Fig. S3 for the effect of surface roughness on droplet growth). The dimple with the smallest magnitude of radius of curvature $\left(\kappa^{-1}=-4.44 \mathrm{~mm}\right.$, Fig. $\left.1(\mathrm{~d})\right)$ shows the smallest condensed water droplets (diameter $\sim 1 \times 10^{2} \mu \mathrm{m}$ ) at the center. In contrast, droplet growth at the apex of bumps is significantly facilitated, which results in larger droplets (diameter $\sim 3 \times 10^{2} \mu \mathrm{m}$ ) compared to droplets on both dimples and flat surfaces.

For a more quantitative analysis to understand these results, the averaged droplet diameter (d) is calculated based on 10 first-generation droplets grown during condensation time $t$ (seconds): $2,400 \leq t \leq 5,400$. Fig. 2 plots the time-evolution of $d$ at the bottom or at the apex of each surface texture for $\kappa^{-1}=13.71 \mathrm{~mm},-13.71 \mathrm{~mm}$, and $-4.44 \mathrm{~mm}$. In the $\log$ - $\log$ plot of the droplet diameter as a function of time shown in Fig. 2, the three topographies display similar slopes close to unity, which is similar to the slope for droplets grown on the flat surface ( $\kappa^{-1} \rightarrow$ infinity). This observation agrees well with previous studies on droplet growth dynamics on flat or microtextured surfaces with different surface wettabilities. ${ }^{9-12,14,15}$

The spatial variation of the averaged droplet diameter $(d)$ has also been studied quantitatively. The dimensionless parameter $p / r$ (see Fig. 3(a) for the geometrical definition of $p$ and $r$ ) is used to define the spatial location of a droplet of interest from the center of bumps and dimples. This dimensionless parameter helps compare the distribution of $d$ with varying $\kappa^{-1}$. Fig. 
PublishiB(s) plots $d$ versus $p / r$ for surfaces with different $\kappa^{-1}$ at $t=5,400 \mathrm{~s}$ (=90 min). $d$ was calculated based on 10 droplets observed on an imaginary circle corresponding to each value of $p / r$. The droplet size at the center of the surface features $\left(d_{0}=d(p / r=0)\right)$ was observed to be greater than that at the far field $\left(d_{\infty}=d(p / r \gg 1)\right)$ for the bump and smaller than $d_{\infty}$ for the two dimples, in agreement with the observation that droplets grow faster on bumps than dimples as shown in Figs. 1 and 2. The droplet diameter $d$ is smallest at the center $(p / r \sim 0)$ for the dimple with a smaller magnitude of $\kappa^{-1}(=-4.44 \mathrm{~mm})$. The two dimples with a negative radius of curvature exhibit similar curves in terms of the spatial location of extrema. Both dimples show that $d$ increases as the location of droplets moves away from the center, then reaches its maximum at $p / r$ $\sim$ 1, which corresponds to the "edge" position as illustrated in Figs. 1(a), 1(b), and 3(a), and finally decreases and converges at the far-field $(p / r>>1)$ corresponding to a flat surface. The spatial distribution of $d$ on the bumpy topography with a positive $\kappa^{-1}$ exhibits the opposite trend: $d$ shows the maximum value at the apex of the bump $(p / r \sim 0)$, then keeps decreasing until it reaches the minimum value at the edge $(p / r \sim 1)$, and finally increases to converge at the far-field.

Since the surface temperature was kept uniform and the effect of surface roughness and the direction of natural convection of humid air is negligible (see additional experimental results shown in Figs. S3 and S4), we attributed this interesting trend in droplet size distribution to the unique effect of millimeter-sized topographical surface features. To test this hypothesis, we first considered the effect of capillary condensation in small cavities, and compared it to the condensation in millimeter-sized concave surface features studied in this work. The effect of micro/nano surface topography and wettability on the actual vapor pressure governing condensation of vapor in the small cavities is described by the Kelvin equation: ${ }^{16-19}$

$$
\ln \frac{P}{P_{0}}=\frac{2 \gamma V_{m}}{R T r_{c}} \cos \theta_{e}
$$


Publishivgere $P$ is the actual vapor pressure, $P_{0}$ is the saturated vapor pressure, $\gamma$ is the interfacial tension between liquid and vapor, $V_{m}$ is the molar volume of the liquid, $R$ is the universal gas constant, $r_{\mathrm{c}}$ is the mean radius of curvature of the liquid-vapor interface, $T$ is the temperature, and $\theta_{e}$ is the equilibrium contact angle. The impact of this capillary condensation effect increases exponentially as the radius of curvature of the surface texture $\left(\kappa^{-1}\right)$ influencing the mean radius of curvature $\left(r_{\mathrm{c}}\right)$ decreases in magnitude. However, in this work, $\left|\kappa^{-1}\right|$ is in the order of millimeters, which is at least two orders of magnitude greater than the length scale where capillary condensation effect is crucial $\left(r_{\mathrm{c}}<10 \mu \mathrm{m}\right) .{ }^{18,19}$ This huge discrepancy implies that a different mechanism may take place.

To explain this suppression of droplet growth on concave features, we postulate that the diffusion flux of water vapor, which was introduced in our previous study to explain the facilitated droplet growth on bumps ${ }^{11}$, may play a key role in controlling the droplet evolution on dimples as well. COMSOL-Multiphysics ${ }^{\circledR}$ was used to simulate the steady state diffusion of water vapor. We employed axisymmetric coordinates for simulating all four bump and dimple cases. The diffusion of water vapor to a surface occurs when the concentration of water vapor near the surface is lower than that far from the surface due to condensation ${ }^{11,12}$. The magnitude of this concentration difference in the simulation was arbitrarily chosen and kept constant because the calculated local diffusion flux near bumps and dimples was further normalized by the diffusion flux on the flat surface far from the bumps and dimples (See Methods in Supplementary Material for more details). The simulated results are presented in Fig. 4, which shows the spatial distribution of the magnitude of the total diffusion flux $\left(J_{\mathrm{C}}\right)$ normalized by the magnitude of the diffusion flux on the nearby flat region $\left(J_{\text {flat }}\right)$ for various bumps and dimples at steady state. The values of the normalized diffusion flux at the far field are the same $\left(J_{\mathrm{C}} / J_{\text {flat }}\right)$ regardless of the surface curvature of bumps/dimples, 
Publishiwgich explains the similar droplet sizes at the far-field $\left(d_{\infty}=d(p / r \gg 1)\right)$ for both dimple and bump cases in Fig. 3(b).

However, within the bumps/dimples, where $p / r<1, J_{\mathrm{C}} / J_{\text {flat }}$ is significantly influenced by $\kappa^{-1}$. For bumps, $J_{\mathrm{C}} / J_{\text {flat }}$ is greater than 1 at the apex, and decreases as $\left|\kappa^{-1}\right|$ increases, while for dimples, $J_{\mathrm{C}} / J_{\text {flat }}$ is less than 1 at the bottom, and increases as $\left|\kappa^{-1}\right|$ increases. This is in good agreement with the observation that droplets become larger on bumps and smaller on dimples as the surfaces become more curved. As $p / r$ increases from 0 to 1 , the spatial distribution of $J_{\mathrm{C}} / J_{\text {flat }}$ from the center to the edge of bumps and dimples shows different patterns. For bumps, $J_{\mathrm{C}} / J_{\text {flat }}$ monotonically decreases from the maximum value at the center until it reaches the minimum value at the edge of bumps as indicated by the color change from red to blue, while it increases with $p / r$ for dimples. This analysis also depicts the reduced and increased values of the $J_{\mathrm{C}} / J_{\text {flat }}$ at the rims of the bumps and dimples, respectively. The spatial distribution of $J_{\mathrm{C}} / J_{\text {flat }}$ on the dimples is consistent with the droplet size distribution shown in Fig. 3(b), implying that the diffusion flux plays a crucial role to cause the unfavorable water condensation on hydrophobic dimples at the millimeter size range.

In summary, we studied the effects of macroscopic surface topography on a dropwise condensation of water. Our results clearly show that the droplets grow faster on a bump than on a dimple, in the millimeter-scale size range. For the same type of surface topography, the facilitation or suppression of condensation becomes more pronounced with a smaller magnitude of the radius of curvature (i.e., when the surface is more curved). The spatial distribution of droplet sizes shows distinct trends for convex and concave features. For bumps, as the dimensionless position of a droplet $(p / r)$ increases, the averaged droplet diameter $d$ decreases from the maximum value at the center to the minimum value at the edge, while for dimples, $d$ increases from the minimum value 
Publishiatgthe center to the maximum value at the edge. This spatial variation of droplet size can be explained by numerical calculation of the diffusion flux of water vapor, which shows its local maximum/minimum value at the bump/dimple center and local minimum/maximum value at the edge as $p / r$ increases. We envision that this macroscopic surface topography effect can be applied to control condensation in a variety of applications such as water harvesting, heat exchangers, and desalination plants.

\section{References}

${ }^{1}$ O. Clus, P. Ortega, M. Muselli, I. Milimouk, and D. Beysens, J. Hydrol. 361, 159-171 (2008).

${ }^{2}$ F. T. Malik, R. M. Clement, D. T. Gethin, W. Krawszik, and A. R. Parker, Bioinspir. Biomim. 9, 031002 (2014).

${ }^{3}$ N. Miljkovic, R. Enright, Y. Nam, K. Lopez, N. Dou, J. Sack, and E. N. Wang, Nano Lett. 13, 179-187 (2013).

${ }^{4}$ J. W. Rose, Proc. Inst. Mech. Eng. A 216, 115-128 (2002).

${ }^{5}$ R. Xiao, N. Miljkovic, R. Enright, and E. N. Wang, Sci. Rep. 3, 1988 (2013).

${ }^{6}$ K. K. Varanasi, M. Hsu, N. Bhate, W. Yang, and T. Deng, Appl. Phys. Lett. 95, 094101 (2009).

${ }^{7}$ L. Mishchenko, M. Khan, J. Aizenberg, and B. D. Hatton, Adv. Funct. Mater. 23, 4577-4584 (2013).

${ }^{8}$ L. Zhai, M. C. Berg, F. C. Cebeci, Y. Kim, J. M. Milwid, M. F. Rubner, and R. E. Cohen, Nano Lett. 6, 1213-1217(2006).

${ }^{9}$ J. B. Boreyko, and C.-H. Chen, Phy. Rev. Lett. 103, 184501 (2009).

${ }^{10}$ K. Zhang, F. Liu, A. J. Williams, X. Qu, J. J. Feng, and C.-H. Chen, Phy. Rev. Lett. 115, 074502 (2015).

${ }^{11}$ K. -C. Park, P. Kim, A. Grinthal, N. He, D. Fox, J. C. Weaver, and J. Aizenberg, Nature. 531, 78-82 (2016).

${ }^{12}$ M.-G. Medici, A. Mongruel, L. Royon, and D. Beysens, Phys. Rev. E 90, 062403 (2014). 
${ }^{14}$ J. L. Viovy, D. Beysens, and C. M. Knobler, Phys. Rev. A 37, 4965-4970 (1988).

${ }^{15}$ D. Beysens, C. R. Phys. 7, 1082-1100 (2006).

${ }^{16}$ L. R. Fisher, R. A. Gamble, and J. Middlehurst, Nature, 290, 575-576 (1981).

${ }^{17}$ M. Qian, and J. Ma, J. Chem. Phys. 130, 214709 (2009).

${ }^{18}$ H. Jo, K. W. Hwang, D. Kim, M. Kiyofumi, H. S. Park, M. H. Kim, and H. S. Ahn, Sci. Rep, 5, 9901 (2015).

${ }^{19}$ H.-J. Butt, K. Graf, and M. Kappl, Physics and Chemistry of Interfaces (WILEY-VCH Verlag GmbH \& Co., Weinheim, 2003) pp.15-20.

\section{Supplementary Material}

See supplementary material for detailed surface fabrication and experimental/calculation methods used in this work and additional information associated with the effect of surface roughness and the direction of gravitational force on condensation of water vapor.

\section{Acknowledgment}

This work was partially supported by the Water Collaboration Seed Funds program of the Northwestern Center for Water Research. 


\section{Publishipgere Captions}

FIG. 1. (a, b) Schematic illustration of the top and cross-sectional views of (a) bump and (b) dimple with the color code for the center (red), edge (green), and flat (purple) regions shown in micrographs. (c-f) Optical micrographs of water droplets grown on surfaces with a radius of curvature of $\kappa^{-1}=$ (c) $4.44 \mathrm{~mm}$, (d) $-4.44 \mathrm{~mm}$, (e) $13.71 \mathrm{~mm}$, and (f) $-13.71 \mathrm{~mm}$ after 2,400 s (= $40 \mathrm{~min}$ ) of condensation (top view). White dashed circles on the main images (shown on the left in the four subfigures) indicate the edge of bumps and dimples. Insets on the right show magnified views of the center, edge, and flat regions indicated by the red, green, and purple frames, respectively, as coded in schematics $(\mathrm{a}, \mathrm{b})$. Yellow circles highlight representative droplets. Scale bars are $2 \mathrm{~mm}$ in the main image (left) and $0.4 \mathrm{~mm}$ in the three magnified images (right) in each subfigure. The flat regions are outside the frame of the main images. It should be also noted that the optical images were taken when the surfaces were vertically positioned. See Supplementary Material (Fig. S4) for more detailed information about the effect of the relative direction of the gravitational force on the droplet size distribution.

FIG. 2. Logarithmic plot of time evolution of averaged droplet diameter $(d)$ grown at the center of hydrophobic millitextured surfaces with various radii of curvature. All the cases show similar slopes close to unity. $d$ is calculated based on 10 droplets on each surface and error bars represent one standard deviation.

FIG. 3. (a) Cross-sectional view of a dimple. $r$ and $p$ represent the maximum radius of a dimple (or a bump) around the axis of symmetry and the distance between a droplet and the axis of symmetry, respectively. (b) The spatial distribution of droplet sizes for textured surfaces with varying $\kappa^{-1}$. At $p / r \cong 0, d$ decreases as $\kappa\left(=1 / \kappa^{-1}\right)$ decreases; while at $p / r \cong 1.67, d$ converges to a value of $\sim 3 \times 10^{2} \mu \mathrm{m}$ regardless of $\kappa^{-1}$. The local extrema at $1 \leq p / r \leq 1.33$ indicate the change of surface curvature near the edge regions, and can be considered as the local dimples at the edge of the bumpy feature or the local bumps at the edge of the dimple, with the corresponding local extrema. $d$ is calculated based on 10 droplets on each surface and error bars represent one standard deviation.

FIG. 4. Magnitude of the total diffusion flux of water vapor $\left(J_{\mathrm{C}}\right)$ for surfaces with $\kappa^{-1}=(\mathrm{a}) 4.4$ $\mathrm{mm}$, (b) $-4.4 \mathrm{~mm}$, (c) $13.7 \mathrm{~mm}$, and (d) $-13.7 \mathrm{~mm}$ normalized by the total diffusion flux on the flat surface far from the bumps and dimples $\left(J_{\text {flat }}\right)$ at steady state. The color gradient from blue to red indicates an increase in the normalized diffusion flux on textured surfaces $\left(J_{\mathrm{C}} / J_{\text {flat }}\right)$ compared to the flat surface indicated by the green color. 


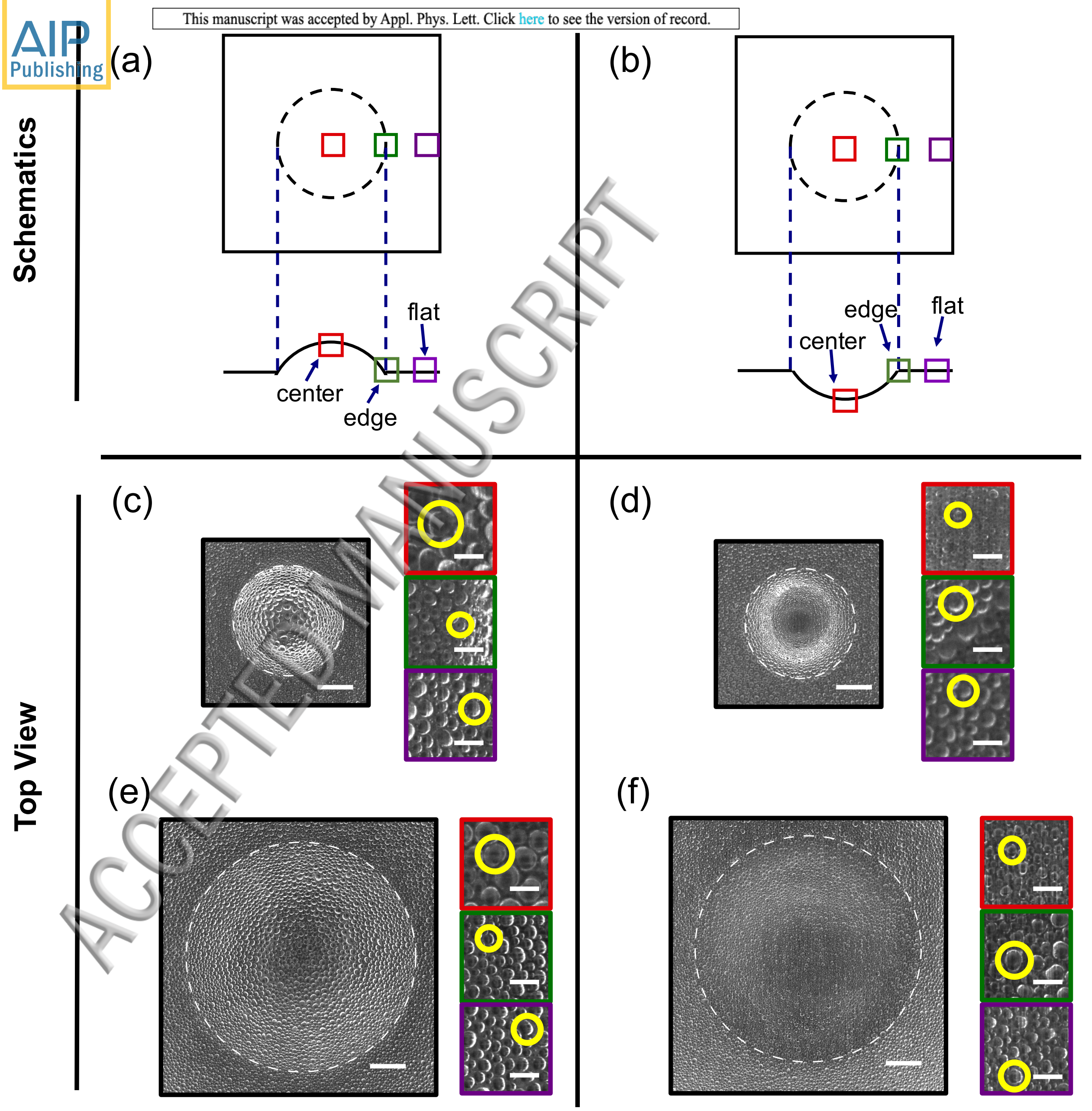




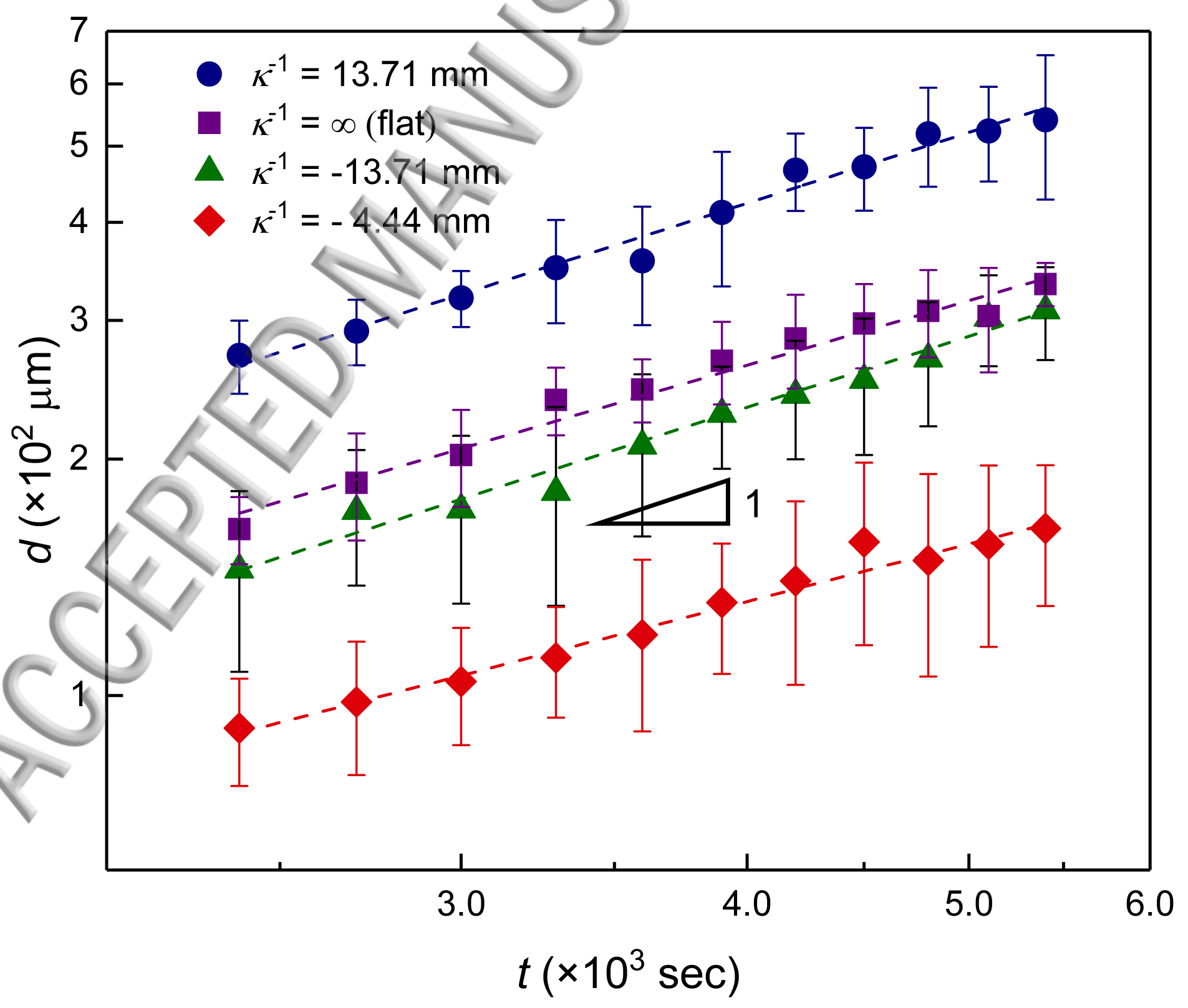


(a)

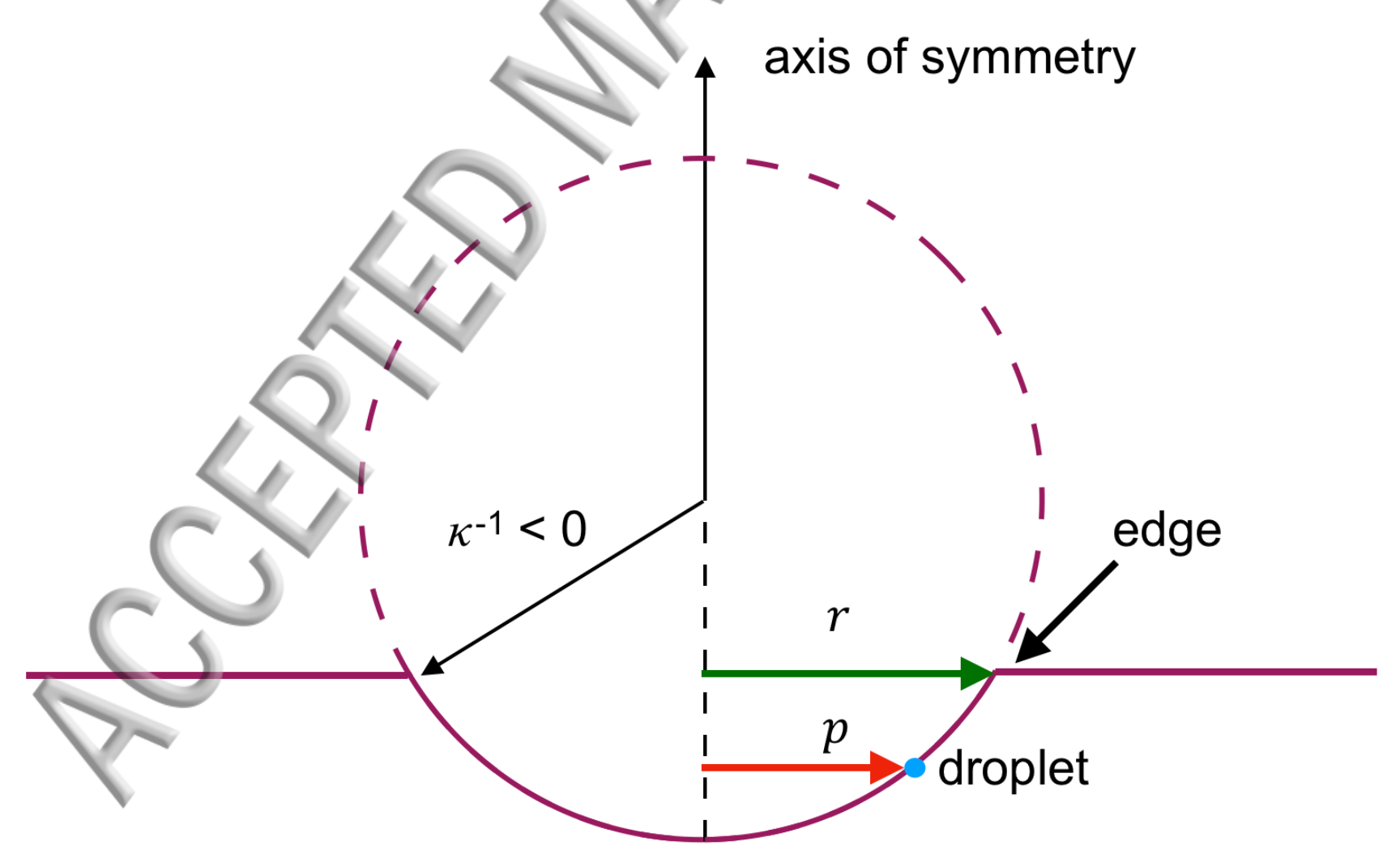

(b)

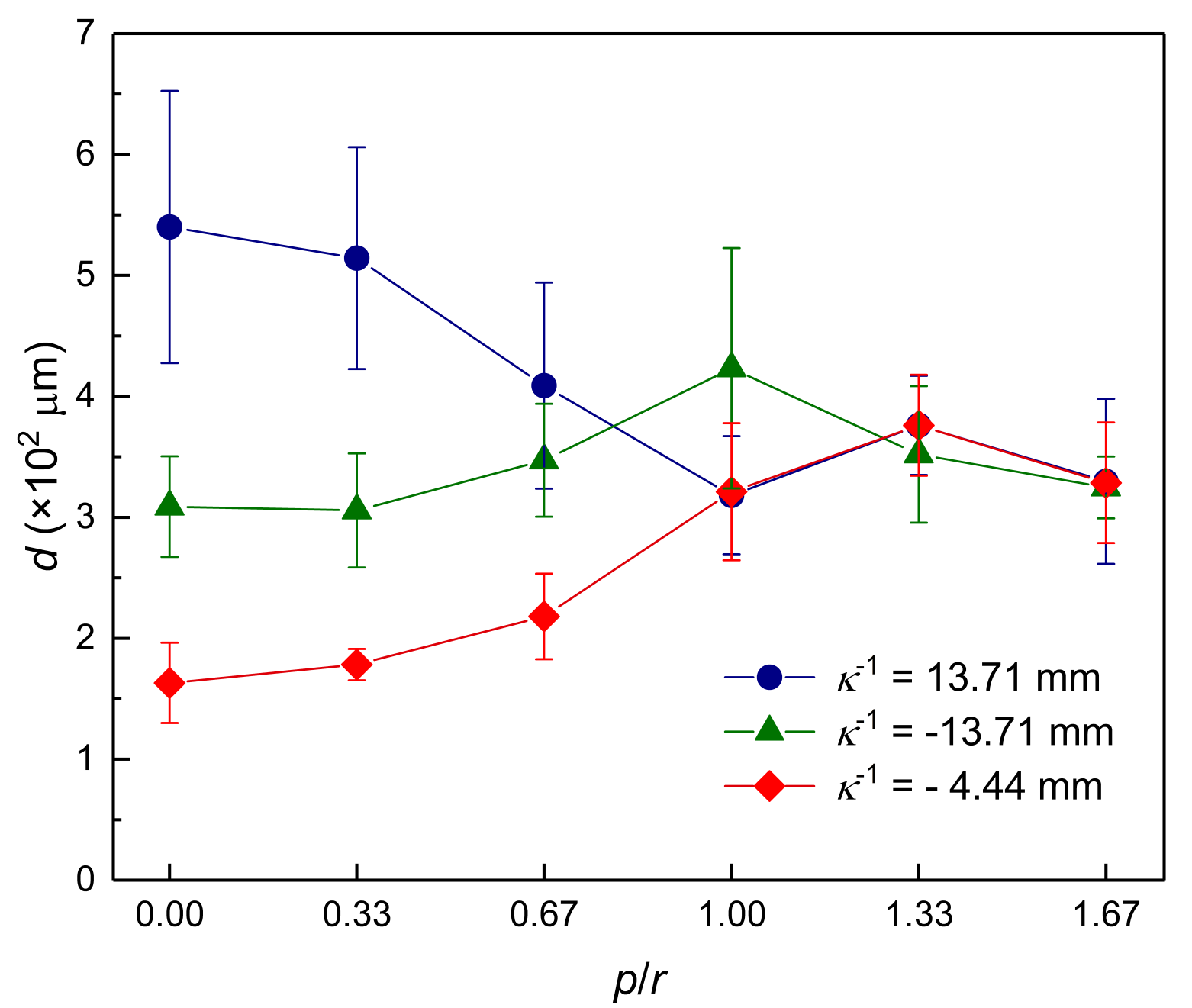


(a)

$$
\kappa^{-1}=4.4 \mathrm{~mm}
$$

(c)

$$
\kappa^{-1}=13.7 \mathrm{~mm}
$$

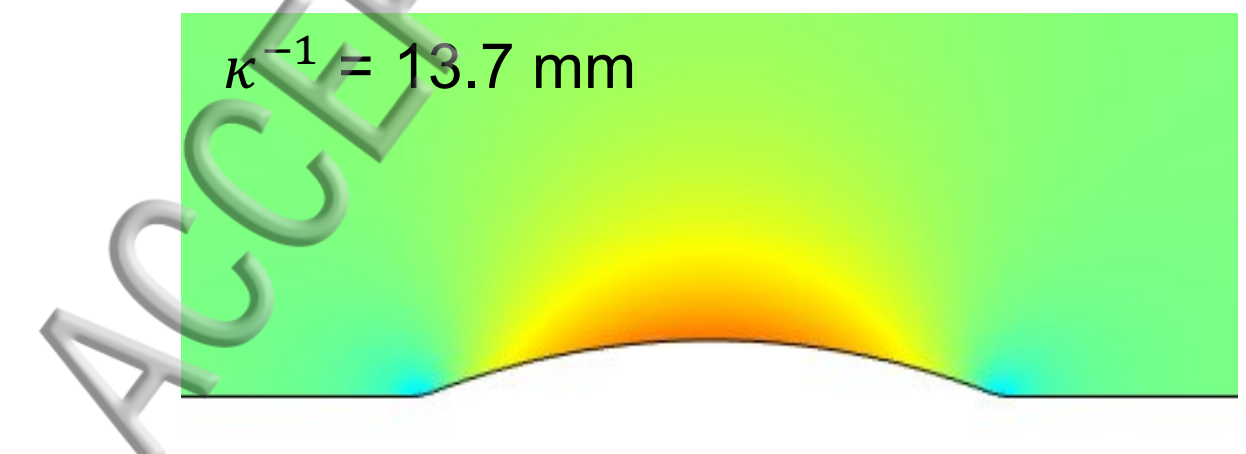

(b)

$$
\kappa^{-1}=-4.4 \mathrm{~mm}
$$

(d)

$$
\kappa^{-1}=-13.7 \mathrm{~mm}
$$

0.2

0.6

1.0

1.4

1.8 